\title{
A Recursive Formulation for Repeated Agency with History Dependence $^{*}$
}

\author{
Ana Fernandes \\ CEMFI \\ Casado del Alisal 5, 28014 Madrid, Spain \\ E-mail: ana.fernandes@cemfi.es \\ and \\ Christopher Phelan \\ Federal Reserve Bank of Minneapolis \\ 90 Hennepin Avenue, Minneapolis, Minnesota 55480 \\ E-mail: cphelan@res.mpls.frb.fed.us
}

\begin{abstract}
We present general recursive methods to handle environments where privately observed variables are linked over time. We show that incentive compatible contracts are implemented recursively with a threat keeping constraint in addition to the usual temporary incentive compatibility and promise keeping conditions. Journal of Economic Literature Classification Numbers: D30, D31, D80, D82.
\end{abstract}

Key Words: Mechanism design, Repeated Agency

\section{INTRODUCTION}

There is now an extensive literature regarding the efficient design of incentive mechanisms in dynamic environments. Included in this literature are models with privately observed endowment shocks (Green [6], Thomas and Worrall [13], Phelan [9], Wang [15], among others), privately observed taste shocks (Atkeson and Lucas [2]) and privately observed effort levels (Spear and Srivastava [11], Phelan and Townsend [10], Phelan [8], Hopen-

* The authors thank Fernando Alvarez, Hugo Hopenhayn, Robert E. Lucas, Jr., the associate editor and two anonymous referees for comments. Fernandes gratefully acknowledges financial support from the Bank of Portugal. The views expressed herein are those of the authors and not necessarily those of the Federal Reserve Bank of Minneapolis or the Federal Reserve System. 
hayn and Nicolini [7], among others). In all of the above examples, there are no exogenous links between periods involving privately observed variables. For instance, in the privately observed endowment and taste shock models, shocks are assumed to be independent over time. In the privately observed effort environments, current variables are functions only of current effort and current (time independent) shocks. Such separations between periods were assumed for the sake of tractability, not realism. ${ }^{1}$

In fact, in most dynamic situations the presence of intertemporal links is overwhelming. Examples include serial correlation in individual income realizations, serial correlation in one's health condition, the fact that the probability of currently finding a job depends on past search effort and the fact that crop output today is a function of the way the soil was treated in previous periods. Given this, relaxing time independence in the context of dynamic agency theory is certainly a pertinent step.

The main simplification one gets by assuming no exogenous links between periods regarding private variables is common knowledge of preferences over continuation contracts. That is, at the beginning of a given date, an agent's forward looking utility of following a given strategy when facing a given contract is independent of past histories - either publicly or privately observed. This makes the definition of incentive compatible contracts from any date onwards a straightforward exercise enabling an equally simple recursive formulation of such problems. Without such an assumption, incentive compatibility is likely to depend on privately observed past histories and this link across time periods turns the recursive formulation into a more involved problem.

The fact that privately observed past histories influence the way in which agents evaluate continuation contracts makes it necessary to consider all the potentially different types of agents that a social planner, say, may be facing at a certain point in time. Incentive compatibility in a recursive sense still has a period by period forward looking component. However, the link across time periods additionally constrains today's decisions, forcing current choices to ensure that no gains are made by an agent who deviated yesterday. In other words, the link across time periods brings an element of threat keeping constraining today's decisions. This additional condition accounts for an enlarged state vector as compared to the case when there is common knowledge of preferences. It also introduces an element of inefficiency to the continuation contract since, in the current period, it is no longer necessary to give incentives towards choices made in the past.

\footnotetext{
${ }^{1}$ Taub [14] allows persistent shocks to private endowments in a linear-quadratic model (with a restriction to linear contracts). Cole and Kocherlakota [4] consider, using nonrecursive methods, a model where agents can unobservably store. Finally, Cole and Kocherlakota [5] consider extending the results of [1] to games where past private play affects future payoffs.
} 
In this paper, we present general recursive methods to handle environments where privately observed variables are linked over time, or where common knowledge of preferences over continuation contracts is violated. We present this first by modifying Green's [6] privately observed endowment model to allow endowments to follow a first-order Markov process. Serial correlation violates common knowledge of preferences over continuation contracts because, at the beginning of any period, the agent alone knows for certain the true probability of future events. We show that this problem nevertheless has a recursive structure and can be solved using a modified Bellman equation formulation. We next present a computed example of our model and discuss the characteristics of the efficient contract. We then extend these methods to two other environments - a taste shock model with Markov shocks and a privately observed effort model where output is a stochastic function of the current effort level and effort in the previous period. We conclude by discussing the applicability of these arguments more generally.

\section{A STOCHASTIC ENDOWMENT MODEL}

In the current section, we will consider a version of Green [6]. Time is discrete and $t=0,1, \ldots, \infty$. Agents in this economy experience endowment shocks which are private information.

For simplicity, let the realizations of the endowment process $h_{t}$ take on two values, $h_{t} \in H=\left\{h_{H}, h_{L}\right\}$, for all $t$, where $h_{H}>h_{L} \geq \underline{c} \in R$. The endowment process of a representative agent follows a first-order Markov process, where $\pi\left(h_{t-1}\right)$ denotes the probability that $h_{t}=h_{H}$ given the previous period realization of the shock. For now, we consider $h_{-1}$, the "seed" value of the Markov process, to be public. We assume $0<\pi\left(h_{j}\right)<1$, $j=H, L$. Denote by $\Pi\left(h_{t+j} \mid h_{t}\right)$ the probability of obtaining endowment realization $h_{t+j}$ given that $h_{t}$ occurred, induced by the Markov process just described. Likewise, let $\Pi\left(h^{t+j} \mid h_{t}\right)$ denote the probability of subsequent history $\left\{h_{t+1}, \ldots, h_{t+j}\right\}$ given $h_{t}$. Endowment realizations are assumed uncorrelated across agents, and all agents face the same probability distribution governing their private endowment process. Further, as in Green [6], we assume that society has the ability to linearly transfer resources through time, and let $q \in(0,1)$ denote the exogenous price of the consumption good in period $t+1$ in terms of date $t$ consumption.

Let $B=[\underline{b}, \bar{b}] \subset R$ be the set of feasible consumption values. Agents evaluate deterministic consumption sequences $c=\left\{c_{t}\right\}_{t=0}^{\infty}$ according to

$$
\bar{U}(c)=\sum_{t=0}^{\infty} \beta^{t} U\left(c_{t}\right),
$$


where $U: B \rightarrow R$ is strictly increasing and strictly concave and $\beta \in(0,1){ }^{2}$ The implied set for the feasible values of momentary utility is $D=[\underline{d}, \bar{d}]$, where $\underline{d}=U(\underline{b})$ and $\bar{d}=U(\bar{b})$.

Efficient allocations in this environment can be found by considering a planning problem where the planner chooses a sequence of conditional transfers to the agent which minimizes the cost of delivering the agent an expected lifetime utility of $w_{0}$, given that endowment probabilities are determined by an initial endowment $h_{-1}$. We also find it useful to consider an auxiliary problem where the planner is additionally constrained to deliver a utility $\hat{w}_{0}$ to an agent whose endowment probabilities are determined by the complement of $h_{-1}$ (denoted $\left.h_{-1}^{c}\right)$ as opposed to $h_{-1}$.

Let $H^{t}$ denote the $t+1$-th Cartesian product of the set $H=\left\{h_{H}, h_{L}\right\}$, with typical element $h^{t}=\left(h_{0}, h_{1}, \ldots, h_{t}\right)$. Define a reporting strategy $\widetilde{h}$ as a sequence $\left\{\widetilde{h}_{t}\left(h^{t}\right)\right\}_{t=0}^{\infty}$ mapping histories $h^{t} \in H^{t}$ to a report of the current endowment realization. Dependence on the initial state $\left(h_{-1}, w_{0}\right)$ is left implicit. Define a transfer system $\tau$ as a sequence of functions $\left\{\tau_{t}\right\}_{t=0}^{\infty}$ such that $\tau_{t}: H^{t} \rightarrow R$ and $\tau_{t}\left(h^{t}\right) \geq-h_{t}$, for all $t$, for all $h^{t} \in H^{t}$. In words, the transfer system states the transfer from the planner to the agent at each date as a function of the history of announcements to that date subject to the planner taking no more from the agent than his announced endowment. ${ }^{3}$ Again, dependence on the initial state is left implicit. In the planner's problem, this initial state is $\left(h_{-1}, w_{0}\right)$, and in the auxiliary planner's problem, this initial state is $\left(h_{-1}, w_{0}, \hat{w}_{0}\right)$.

The timing of events is as follows. Period $t$ begins after history $h^{t-1} \in$ $H^{t-1}$ has taken place and the sequence of reports $\widetilde{h}^{t-1}\left(h^{t-1}\right)=\left(\widetilde{h}_{0}\left(h_{0}\right)\right.$, $\left.\widetilde{h}_{1}\left(h^{1}\right), \ldots, \widetilde{h}_{t-1}\left(h^{t-1}\right)\right)$ has been submitted to the planner. The agent observes his period $t$ income shock $h_{t}$ and reports its realization to the planner according to the strategy $\widetilde{h}_{t}\left(h^{t}\right)$. The planner then provides the (possibly negative) transfer $\tau_{t}\left(\widetilde{h}^{t}\right)$ which leaves the agent with current utility of $U\left(h_{t}+\tau_{t}\left(\widetilde{h}^{t}\left(h^{t}\right)\right)\right)$.

\footnotetext{
${ }^{2}$ The restriction of the consumption set to a compact interval has the sole purpose of ensuring the boundedness of expected discounted utility and may therefore be substantially relaxed.

${ }^{3}$ Imposing $\tau\left(h_{t}\right) \geq-h_{t}$ does not rule out the possibility, for reported endowment $\tilde{h}_{t}$ and actual endowment $h_{t}$, that $\tau\left(\tilde{h}_{t}\right)+h_{t} \leq \underline{b}$, where $\underline{b}$ is the lower bound on consumption. Given this, one must either define $U$ over consumption levels lower than $\underline{b}$ or disallow the agent from claiming a higher than actual endowment. If one assumes $\bar{U}(c)=-\infty$, for $c \in(-\infty, \underline{b})$ these are equivalent. A contract, at arbitrarily low cost, can keep an agent with a low endowment from reporting high by having an arbitrarily small probability of a low-claiming-high agent consuming below $\underline{b}$. For notational convenience, we do not impose a restriction that low agents cannot claim high. In our computed example, this restriction is explicit.
} 
In what follows, it will be convenient to map the transfers $\tau$ into a sequence of utility endowments. Let $C(\widetilde{u})$ denote the amount of the consumption good needed to provide $\widetilde{u}$ units of momentary utility to a representative agent. From the properties of the function $U$, we have that $C(\widetilde{u}) \equiv U^{-1}(\widetilde{u})$ is uniquely defined for all $\widetilde{u} \in D$ and $C: D \rightarrow B$ is a strictly increasing and strictly convex function. In the planner's problem, we define an allocation $u$ to be a sequence of functions $\left\{u_{t}\right\}_{t=0}^{\infty}$ such that $u_{t}: H^{t} \rightarrow D$, where

$$
C\left(u_{t}\left(\widetilde{h}^{t}\right)\right)-\widetilde{h}_{t}=\tau_{t}\left(\widetilde{h}^{t}\right)
$$

We will now more fully describe the infinite sequence problem and the infinite sequence auxiliary problem faced by the planner. As stated earlier, in the planner's problem the planner takes as given values for $\left(h_{-1}, w_{0}\right)$. We say an allocation is incentive compatible if it induces an agent with initial condition $h_{-1}$ never to misreport his endowment realization. An incentive compatible allocation $u$ satisfies, for all reporting strategies $\widetilde{h}$,

$$
\begin{gathered}
\sum_{t=0}^{\infty} \sum_{h^{t} \in H^{t}} \beta^{t} u_{t}\left(h^{t}\right) \Pi\left(h^{t} \mid h_{-1}\right) \\
\geq \sum_{t=0}^{\infty} \sum_{h^{t} \in H^{t}} \beta^{t} U\left(h_{t}+C\left(u_{t}\left(\widetilde{h}^{t}\left(h^{t}\right)\right)\right)-\widetilde{h}_{t}\left(h^{t}\right)\right) \Pi\left(h^{t} \mid h_{-1}\right) .
\end{gathered}
$$

An allocation satisfies promise keeping if it delivers $w_{0}$ units of expected discounted utility to an agent with initial history $h_{-1}$ :

$$
w_{0}=\sum_{t=0}^{\infty} \sum_{h^{t} \in H^{t}} \beta^{t} u_{t}\left(h^{t}\right) \Pi\left(h^{t} \mid h_{-1}\right)
$$

Given initial state variables $\left(h_{-1}, w_{0}\right)$, the planner's problem is defined as choosing an allocation $u$ to minimize the cost of providing $w_{0}$ units of expected discounted utility,

$$
V\left(h_{-1}, w_{0}\right) \equiv \min _{u}\left\{\sum_{t=0}^{\infty} \sum_{h^{t} \in H^{t}} q^{t}\left[C\left(u_{t}\left(h^{t}\right)\right)-h_{t}\right] \Pi\left(h^{t} \mid h_{-1}\right)\right\}
$$

subject to (1) and (2).

We define the auxiliary planning problem as that of choosing an allocation to minimize the cost of providing $\left(w_{0}, \hat{w}_{0}\right)$ units of expected discounted 
utility to agents with seed values $\left(h_{-1}, h_{-1}^{c}\right)$, respectively:

$$
V_{A}\left(h_{-1}, w_{0}, \hat{w}_{0}\right) \equiv \min _{u}\left\{\sum_{t=0}^{\infty} \sum_{h^{t} \in H^{t}} q^{t}\left[C\left(u_{t}\left(h^{t}\right)\right)-h_{t}\right] \Pi\left(h^{t} \mid h_{-1}\right)\right\}
$$

subject to (1), (2), as well as

$$
\hat{w}_{0}=\sum_{t=0}^{\infty} \sum_{h^{t} \in H^{t}} \beta^{t} u_{t}\left(h^{t}\right) \Pi\left(h^{t} \mid h_{-1}^{c}\right)
$$

Let $W^{*}\left(h_{-1}\right)$ be the set of $\left(w_{0}, \hat{w}_{0}\right)$ values such that there exists an allocation satisfying (1), (2), and (5). For now, we simply assume (and later prove) that this is a non-empty, compact set. Finally, note that solving the auxiliary problem is one method of solving the planning problem. That is,

$$
V\left(h_{-1}, w_{0}\right)=\min _{\hat{w}_{0}} V_{A}\left(h_{-1}, w_{0}, \hat{w}_{0}\right)
$$

subject to

$$
\left(w_{0}, \hat{w}_{0}\right) \in W^{*}\left(h_{-1}\right)
$$

We next turn to showing that the auxiliary problem is recursive.

\subsection{The Recursive Formulation}

This subsection provides a recursive formulation for the auxiliary planner's problem described above. Our strategy is to reformulate the auxiliary planner's problem into the form of Stokey and Lucas [12] and, from there, simply apply their theorems. In their formulation, the only choice variables are the future values of the state variables, and the constraint set is expressed recursively. That is, if $s_{t}$ is the state variable and $h_{t}$ is the current shock, the per-period, certain-outcome objective function is expressed as $F\left(h_{t}, s_{t}, s_{t+1}\right)$ and the constraint set is expressed, for all $t$ and histories of shocks $h^{t}$, as $s_{t+1}\left(h^{t}\right) \in \Gamma\left(s_{t}\left(h^{t-1}\right), h_{t}\right)$. The major result of this section is to show that the constraint set of the auxiliary planner's problem can be expressed in this form.

To do this, first take as given an allocation (or plan) $\left\{u_{t}\left(h^{t}\right)\right\}_{t=0}^{\infty}$. For such a plan, let

$$
w_{t+1}\left(h^{t}\right) \equiv \sum_{\tau=0}^{\infty} \sum_{h^{\tau} \in H^{\tau}} \beta^{\tau} u_{t+1+\tau}\left(h^{t}, h^{\tau}\right) \Pi\left(h^{\tau} \mid h_{t}\right)
$$


and

$$
\hat{w}_{t+1}\left(h^{t}\right) \equiv \sum_{\tau=0}^{\infty} \sum_{h^{\tau} \in H^{\tau}} \beta^{\tau} u_{t+1+\tau}\left(h^{t}, h^{\tau}\right) \Pi\left(h^{\tau} \mid h_{t}^{c}\right) .
$$

The first object represents the forward looking lifetime utility of an agent at the beginning of date $t+1$, given the above plan, after the truthful announcement of endowment $h_{t}$ and truthful revelation of subsequent shocks. The second object represents the forward looking lifetime utility of an agent at the beginning of date $t+1$, given the above plan, where $h_{t}$ was announced but $h_{t}^{c}$ actually occurred, again given truthful revelation of subsequent shocks.

We next prove the following lemma which shows that if an allocation $u$ satisfies incentive compatibility, then an agent who has lied in the past will weakly prefer to tell the truth in the future. To do this, let $\left\{\widetilde{h}_{\tau}\left(h^{\tau} \mid h^{t-1}\right.\right.$, $\left.\left.\hat{h}^{t-1}\right)\right\}_{\tau=0}^{\infty}$ denote a continuation reporting strategy for dates $t+\tau$ on, after history $h^{t-1}$ and announced history $\hat{h}^{t-1}$. We define an allocation $u$ to be incentive compatible after $\left(h^{t-1}, \hat{h}^{t-1}\right)$ if, for all $\left\{\widetilde{h}_{\tau}\left(h^{\tau} \mid h^{t-1}, \hat{h}^{t-1}\right)\right\}_{\tau=0}^{\infty}$,

$$
\begin{gathered}
\sum_{\tau=0}^{\infty} \sum_{h^{\tau} \in H^{\tau}} \beta^{\tau} u_{t+\tau}\left(\hat{h}^{t-1}, h^{\tau}\right) \Pi\left(h^{\tau} \mid h_{t-1}\right) \geq \\
\sum_{\tau=0}^{\infty} \sum_{h^{\tau} \in H^{\tau}} \beta^{\tau} U\left(h_{\tau}+C\left(u_{t+\tau}\left(\hat{h}^{t-1}, \widetilde{h}^{\tau}\left(h^{\tau} \mid h^{t-1}, \hat{h}^{t-1}\right)\right)\right)-\right. \\
\left.\widetilde{h}_{\tau}\left(h^{\tau} \mid h^{t-1}, \hat{h}^{t-1}\right)\right) \Pi\left(h^{\tau} \mid h_{t-1}\right) .
\end{gathered}
$$

LEMMA 2.1. Let u satisfy equation (1), the time-zero incentive compatibility condition. Then, for any time period $t$, past history of reports $\hat{h}^{t-1}$ and true realizations $h^{t-1}, u$ is incentive compatible after $\left(h^{t-1}, \hat{h}^{t-1}\right)$.

Proof. The incentive compatibility constraint, equation (1), implies that $u$ is incentive compatible after any truthfully reported history $h^{t-1}$. If not, there exists a continuation strategy $\widetilde{h}=\left\{\widetilde{h}_{\tau}\left(h^{\tau} \mid h^{t-1}, h^{t-1}\right)\right\}_{\tau=0}^{\infty}$ which delivers a higher continuation utility than truth-telling. Consider a time zero reporting strategy which consists of truth-telling before date $t$ and after all histories other than $h^{t-1}$ but which follows $\widetilde{h}$ after history $h^{t-1}$. Since $h^{t-1}$ occurs with positive probability, this time zero reporting strategy dominates truth-telling as well, contradicting (1). This implies

$$
\sum_{\tau=0}^{\infty} \sum_{h^{\tau} \in H^{\tau}} \beta^{\tau} u_{t+\tau}\left(h^{t-1}, h^{\tau}\right) \Pi\left(h^{\tau} \mid h_{t-1}\right) \geq
$$




$$
\begin{gathered}
\sum_{\tau=0}^{\infty} \sum_{h^{\tau} \in H^{\tau}} \beta^{\tau} U\left(h_{\tau}+C\left(u_{t+\tau}\left(h^{t-1}, \widetilde{h}^{\tau}\left(h^{\tau} \mid h^{t-1}, h^{t-1}\right)\right)\right)-\right. \\
\left.\widetilde{h}_{\tau}\left(h^{\tau} \mid h^{t-1}, h^{t-1}\right)\right) \Pi\left(h^{t+\tau} \mid h_{t-1}\right),
\end{gathered}
$$

for all continuation reporting strategies $\widetilde{h}=\left\{\widetilde{h}_{\tau}\left(h^{\tau} \mid h^{t-1}, h^{t-1}\right)\right\}_{\tau=0}^{\infty}$.

Consider $\hat{h}^{t-1} \neq h^{t-1}$. We need to show, for all $\widetilde{h}=\left\{\widetilde{h}_{\tau}\left(h^{\tau} \mid h^{t}, \hat{h}^{t}\right)\right\}_{\tau=0}^{\infty}$,

$$
\begin{gathered}
\sum_{\tau=0}^{\infty} \sum_{h^{\tau} \in H^{\tau}} \beta^{\tau} u_{t+\tau}\left(\hat{h}^{t-1}, h^{\tau}\right) \Pi\left(h^{\tau} \mid h_{t-1}\right) \geq \\
\sum_{\tau=0}^{\infty} \sum_{h^{\tau} \in H^{\tau}} \beta^{\tau} u\left(h_{\tau}+C\left(u_{t+\tau}\left(\hat{h}^{t-1}, \widetilde{h}^{\tau}\left(h^{\tau} \mid h^{t-1}, \hat{h}^{t-1}\right)\right)\right)-\right. \\
\left.\widetilde{h}_{\tau}\left(h^{\tau} \mid h^{t-1}, \hat{h}^{t-1}\right)\right) \Pi\left(h^{t+\tau} \mid h_{t-1}\right) .
\end{gathered}
$$

Suppose there exists a history, an announced history profile $\left(h^{t-1}, \hat{h}^{t-1}\right)$, and a reporting strategy $\widetilde{h}$ after $\left(h^{t-1}, \hat{h}^{t-1}\right)$ which does better than truthtelling (or violates equation (12)). For this to be true, it must be the case that conditional on either $h_{t}=h_{H}$ or $h_{t}=h_{L}$, the reporting strategy improves on truth-telling. For now, let this be $h_{t}=h_{H}$. Consider another reporting strategy $\bar{h}$ which follows $\widetilde{h}$ given $h_{t}=h_{H}$ and involves truth-telling following $h_{t}=h_{L}$. Since $h_{H}$ occurs with positive probability, $\bar{h}$ also dominates truth-telling. Next, consider the history and the announced history profile $\left(\hat{h}^{t-1}, \hat{h}^{t-1}\right)$. This profile invokes the same continuation plan $u_{t}$ as $\left(h^{t-1}, \hat{h}^{t-1}\right)$ (since the announced history is the same) but involves different probabilities of future events (since the actual histories differ). Nevertheless, given the actual history $\hat{h}^{t-1}$, event $h_{t}=h_{H}$ still occurs with positive probability, and thus if $\bar{h}$ dominates truth-telling given profile $\left(h^{t-1}, \hat{h}^{t-1}\right)$, it dominates truth-telling given profile $\left(\hat{h}^{t-1}, \hat{h}^{t-1}\right)$. This contradicts equation (11). The same argument is made if $\widetilde{h}$ improves on truth-telling given $h_{t}=h_{L}$.

We now turn to the main result of this section.

THEOREM 2.1. Let $u=\left\{u_{t}\right\}_{t=0}^{\infty}$ be a sequence of functions such that $u_{t}: H^{t} \rightarrow D$. The sequence $u$ is incentive compatible (or satisfies 1 ) if and only if it satisfies the following condition, for all time periods t, and all histories $h^{t}$ :

$$
u_{t}\left(h^{t}\right)+\beta w_{t+1}\left(h^{t}\right) \geq
$$




$$
U\left(h_{t}+C\left(u_{t}\left(h^{t-1}, h_{t}^{c}\right)\right)-h_{t}^{c}\right)+\beta \hat{w}_{t+1}\left(h^{t-1}, h_{t}^{c}\right) .
$$

Proof. Suppose a sequence $u$ satisfies (1) but for some $t$ and $h^{t}$ violates (13). Given this, define a deviation strategy $\tilde{h}$ in which the agent lies only at date $t$ given $h^{t}$. Since (13) is violated, and all histories occur with positive probability, (1) is violated.

Next, suppose $u$ satisfies (13) for all $t$ and $h^{t}$, but violates (1). Then there exists a reporting strategy $\widetilde{h}$ such that

$$
\begin{gathered}
W_{0}(\widetilde{h}) \equiv \\
\sum_{t=0}^{\infty} \sum_{h^{t} \in H^{t}} \beta^{t} U\left(h_{t}+C\left(u_{t}\left(\widetilde{h}^{t}\left(h^{t}\right)\right)\right)-\widetilde{h}_{t}\left(h^{t}\right)\right) \Pi\left(h^{t} \mid h_{-1}\right)
\end{gathered}
$$

exceeds $w_{0}$, the utility associated with truth-telling, by an amount $a>0$.

Define the strategy $\widetilde{h}^{\tau}$ as follows. Let $\widetilde{h}_{t}^{\tau}\left(h^{t}\right)=\widetilde{h}_{t}\left(h^{t}\right)$, if $t<\tau$, and $\widetilde{h}_{t}^{\tau}\left(h^{t}\right)=h_{t}$, for $t \geq \tau$. Define $H_{\text {true }}^{t}=\left\{h^{t} \mid \widetilde{h}_{t}\left(h^{t}\right)=h_{t}\right\}$ and $H_{\text {false }}^{t}=$ $\left\{h^{t} \mid \widetilde{h}_{t}\left(h^{t}\right)=h_{t}^{c}\right\}$. We then have

$$
\begin{aligned}
W_{0}\left(\widetilde{h}^{\tau}\right)= & \sum_{t=0}^{\tau} \sum_{h^{t} \in H^{t}} \beta^{t} u\left(h_{t}+C\left(u_{t}\left(\widetilde{h}^{t}\left(h^{t}\right)\right)\right)-\widetilde{h}_{t}\right) \Pi\left(h^{t} \mid h_{-1}\right) \\
& +\beta^{\tau+1}\left\{\sum_{h^{\tau} \in H_{\text {true }}^{\tau}} w_{\tau+1}\left(\widetilde{h}^{\tau}\left(h^{\tau}\right)\right) \Pi\left(h^{\tau} \mid h_{-1}\right)+\right. \\
& \left.\sum_{h^{\tau} \in H_{\text {false }}^{\tau}} \hat{w}_{\tau+1}\left(\widetilde{h}^{\tau}\left(h^{\tau}\right)\right) \Pi\left(h^{\tau} \mid h_{-1}\right)\right\}
\end{aligned}
$$

Since the set of utilities $D$ is bounded and $\beta<1, W_{0}\left(\tilde{h}^{\tau}\right)$ converges to $W_{0}(\tilde{h})$ as $\tau \rightarrow \infty$. Thus we can choose $\tau$ such that $\left|W_{0}(\widetilde{h})-W_{0}\left(\tilde{h}^{\tau}\right)\right|<$ $a / 2$. We next show $W_{0}\left(\widetilde{h}^{\tau}\right) \leq w_{0}$, yielding a contradiction.

Consider date $\tau$, the last period where $\widetilde{h}^{\tau}$ can recommend lying and suppose $\widetilde{h}^{\tau}\left(h^{\tau-1}\right)=h_{\tau-1}$ (truth-telling at date $\left.\tau-1\right)$. That equation (13) holds in period $\tau$ implies that $\widetilde{h}^{\tau}$ does not improve on truth-telling from pe$\operatorname{riod} \tau$ on. Lemma 2.1 then implies that $\widetilde{h}^{\tau}$ does not improve on truth-telling from period $\tau$ on in the case that the agent lied at date $\tau-1$. Given this, that (13) holds for all $t<\tau$ implies (through backward application) that $\tilde{h}^{\tau}\left(h^{t}\right)$ does not improve on truth-telling at date $t=0$. This contradicts the assumption that $W_{0}(\widetilde{h})-w_{0}>a$. 
Equation (13) represents the temporary incentive compatibility constraint, to use the terminology of Green [6]. The consequences of lying imply a different immediate utility as well as a different continuation promise relative to the transfer and continuation promise obtained under the true report. Unlike the case where preferences over future contracts are common knowledge, the planner now potentially faces two types of agents: those who received endowment $h_{t}$ and reported so, and those who had endowment $h_{t}^{c}$ yet reported $h_{t}$. Again, unlike the common knowledge benchmark, these two agents are different since they compute the expectation of future utility streams using different probability distributions. Ensuring that incentive compatibility holds implies therefore that both continuation promised utilities $-w_{t+1}$ and $\hat{w}_{t+1}$ - have to be delivered, in period $t+1$. This is carried out by ensuring that the utility endowments $\left\{u_{t+1+j}\left(h^{t}, h^{j}\right)\right\}_{j=0}^{\infty}$ deliver utility $w_{t+1}\left(h^{t}\right)$ under probability distribution $\Pi\left(h^{j} \mid h_{t}\right)$ and that they simultaneously deliver utility $\hat{w}_{t+1}\left(h^{t}\right)$ under $\Pi\left(h^{j} \mid h_{t}^{c}\right)$. We refer to this latter constraint as threat keeping, whereas the former condition is usually referred to as promise keeping.

Since constraining $\left\{u_{t+1+j}\left(h^{t}, h^{j}\right)\right\}_{j=0}^{\infty}$ to deliver $\hat{w}_{t+1}\left(h^{t}\right)$ under $\Pi\left(h^{j} \mid\right.$ $\left.h_{t}^{c}\right)$ has the purpose of making the sequence $\left\{u_{t}\left(h^{t}\right), u_{t+1+j}\left(h^{t}, h^{j}\right)\right\}_{j=0}^{\infty}$ incentive compatible from the perspective of period $t$ (that is, it ensures that an agent who lied at time $t$ does not benefit from such deviation as his continuation utility $\hat{w}_{t+1}\left(h^{t}\right)$ is held constant), it introduces an element of inefficiency concerning future contracts: both principal and agent could be made better off if such an adding up constraint was not present.

We now turn to writing the constraint set in the recursive form $s_{t+1}\left(h^{t}\right) \in$ $\Gamma\left(h_{t}, s_{t}\left(h^{t-1}\right)\right)$. From the definitions (8) and (9), one can write

$$
w_{t}\left(h^{t-1}\right)=\sum_{h_{t}}\left\{u_{t}\left(h^{t}\right)+\beta w_{t+1}\left(h^{t}\right)\right\} \Pi\left(h_{t} \mid h_{t-1}\right),
$$

and

$$
\hat{w}_{t}\left(h^{t-1}\right)=\sum_{h_{t}}\left\{u_{t}\left(h^{t}\right)+\beta w_{t+1}\left(h^{t}\right)\right\} \Pi\left(h_{t} \mid h_{t-1}^{c}\right) .
$$

Likewise, if equations (16) and (17) hold for every $h^{t-1}$, then equations (8) and (9) hold.

For given values of $t$, and $h^{t-1}$, the two equations (16) and (17) can be solved for $u_{t}\left(h^{t-1}, h_{L}\right)$ and $u_{t}\left(h^{t-1}, h_{H}\right)$ as linear functions of $w_{t}\left(h^{t-1}\right)$, $\hat{w}_{t}\left(h^{t-1}\right), w_{t+1}\left(h^{t}\right)$ and $\hat{w}_{t+1}\left(h^{t}\right)$. This implies one can consider the choice variables to be a sequence of conditional lifetime continuation utilities $w=$ $\left\{w_{t}\left(h^{t-1}\right), \hat{w}_{t}\left(h^{t-1}\right)\right\}_{t=1}^{\infty}$ such that equations (16) and (17) hold, instead of the sequence of utility payments $u=\left\{u_{t}\left(h^{t}\right)\right\}_{t=0}^{\infty}$. Any sequence of 
conditional lifetime continuation utilities $w$ uniquely implies, for a given $s_{0}=\left\{h_{-1}, w_{0}, \hat{w}_{0}\right\}$, a sequence of conditional utility payments $u$, and any sequence of conditional utility payments $u$ can be achieved by a sequence of continuation utilities $w$. Theorem 1 in turn implies that if promise keeping and threat keeping hold — (16) and (17), respectively - along with (13) - the temporary incentive compatibility constraint - then the sequence $\left\{w_{t}\left(h^{t-1}\right), \hat{w}_{t}\left(h^{t-1}\right)\right\}_{t=1}^{\infty}$ is incentive compatible. Thus conditions (16), (17), and (13) imply the same constraint set for the auxiliary planning problem as equations (2), (5) and (1), but where each constraint is written only as a function of $s_{t}$ and $s_{t+1}$, for

$$
s_{t}\left(h^{t-1}\right) \equiv\left(h_{t-1}, w_{t}\left(h^{t-1}\right), \hat{w}_{t}\left(h^{t-1}\right)\right) .
$$

Finally, the return function for the auxiliary planner's problem can be stated

$$
F\left(h_{t}, s_{t}\left(h^{t-1}\right), s_{t+1}\left(h^{t}\right)\right)=C\left(u_{t}\left(h^{t}\right)\right)-h_{t},
$$

where $u_{t}\left(h^{t}\right)$ is determined by $s_{t}\left(h^{t-1}\right)$ and $s_{t+1}\left(h^{t}\right)$ according to (16) and (17). This puts our problem in the form of Stokey and Lucas [12] with the following exception. We have not yet shown that the set of $(w, \hat{w})$ pairs such that there exists an incentive compatible contract which delivers these lifetime utilities is non-empty and compact. This is constructively shown using a rather straightforward application of Abreu, Pearce, and Stacchetti [1].

Let $W^{*}\left(h_{-}\right)$be the set of $(w, \hat{w})$ pairs such that there exists an allocation satisfying (1), (2), and (5).

Lemma 2.2. The set $W^{*}\left(h_{-}\right)$is non-empty and compact for each $h_{-}$.

Proof. Setting $u_{t}=U(\bar{b})$ at all dates given all announcements is incentive compatible and delivers the same utility regardless of $h_{-}$, thus $W^{*}\left(h_{-}\right)$ is non-empty. Next, for each $h_{-}$, for an arbitrary compact set $W\left(h_{-}\right)$, define

$$
\begin{gathered}
B(W)\left(h_{-}\right)=\left\{(w, \hat{w}) \mid \text { there exists }\left(u(h), w^{\prime}(h), \hat{w}^{\prime}(h)\right)\right. \text { such that } \\
u(h) \in[U(\underline{b}), U(\bar{b})] \text { for each } h \\
\left(w^{\prime}(h), \hat{w}^{\prime}(h)\right) \in W(h) \text { for each } h \\
w=\sum_{h \in H}\left\{u(h)+\beta w^{\prime}(h)\right\} \Pi\left(h \mid h_{-}\right)
\end{gathered}
$$




$$
\hat{w}=\sum_{h \in H}\left\{u(h)+\beta w^{\prime}(h)\right\} \Pi\left(h \mid h_{-}^{c}\right),
$$

and

$$
\left.u(h)+\beta w^{\prime}(h) \geq U\left(h+C\left(u\left(h^{c}\right)\right)-h^{c}\right)+\beta \hat{w}^{\prime}\left(h^{c}\right)\right\} .
$$

This is the standard Abreu, Pearce and Stacchetti operator applied to this contracting framework. Results from [1] apply, delivering that $W^{*}\left(h_{-}\right)$is compact.

Given the state space defined by $\left(h_{-}, W^{*}\left(h_{-}\right)\right)$, we can write the auxiliary planner's problem in the following recursive form. In this form, for each $h \in H$, the choice variables are $u(h)$, the current utility transfer as a function of the announced endowment; $w^{\prime}(h)$, the lifetime continuation utility promise as a function of the announced endowment; and $\hat{w}^{\prime}(h)$, the utility that the planner tells the agent he will receive from the continuation allocation if he announces $h$ but is lying. We write the state variables as $h_{-}$, the reported endowment realization last period; $w$, the promised utility of an agent who truthfully announced $h_{-}$last period; and $\hat{w}$, the promised utility of an agent who falsely announced $h_{-}$last period. Our recursive operator $T$, defined on the space of bounded continuous functions mapping triples $\left(h_{-}, w, \hat{w}\right) \rightarrow R$, is then

$$
\begin{gathered}
T\left(v_{A}\right)\left(h_{-}, w, \hat{w}\right) \equiv \\
\min _{u(h), w^{\prime}(h), \hat{w}^{\prime}(h)} \sum_{h \in H}\left\{C(u(h))-h+q v_{A}\left(h, w^{\prime}(h), \hat{w}^{\prime}(h)\right)\right\} \Pi\left(h \mid h_{-}\right)
\end{gathered}
$$

subject to

$$
\begin{aligned}
& w=\sum_{h \in H}\left\{u(h)+\beta w^{\prime}(h)\right\} \Pi\left(h \mid h_{-}\right), \\
& \hat{w}=\sum_{h \in H}\left\{u(h)+\beta w^{\prime}(h)\right\} \Pi\left(h \mid h_{-}^{c}\right)
\end{aligned}
$$

and, for each $h \in H$,

$$
u(h)+\beta w^{\prime}(h) \geq U\left(h+C\left(u\left(h^{c}\right)\right)-h^{c}\right)+\beta \hat{w}^{\prime}\left(h^{c}\right),
$$

along with $u(h) \in[U(\underline{b}), U(\bar{b})]$ and $\left(w^{\prime}(h), \hat{w}^{\prime}(h)\right) \in W^{*}(h)$. The results of Stokey and Lucas [12] for this problem can then be summarized by the following Lemma. 
Lemma 2.3. The function $V_{A}$ is the unique fixed point of T. Further, the policies $u\left(h \mid h_{-}, w, \hat{w}\right), w^{\prime}\left(h \mid h_{-}, w, \hat{w}\right), \hat{w}^{\prime}\left(h \mid h_{-}, w, \hat{w}\right)$ which solve (21) solve the infinite sequence auxiliary planning problem. Conversely, a solution $\left\{u_{t}\left(h^{t}\right)\right\}_{t=0}^{\infty}$ to the infinite sequence auxiliary planning problem solves

$$
\begin{gathered}
V_{A}\left(h_{t-1}, w_{t}\left(h^{t-1}\right), \hat{w}_{t}\left(h^{t-1}\right)\right)= \\
\sum_{h_{t} \in H}\left\{C\left(u_{t}\left(h^{t}\right)\right)-h_{t}+q V_{A}\left(h_{t}, w_{t+1}^{\prime}\left(h^{t}\right), \hat{w}_{t+1}^{\prime}\left(h^{t}\right)\right)\right\} \Pi\left(h_{t} \mid h_{t-1}\right)
\end{gathered}
$$

for all $t-1, h^{t-1}$.

Proof. $\quad$ Stokey and Lucas [12], Theorems 4.2, 4.3, 4.4, 4.5.

\subsection{Discussion}

We now look more carefully into the relationship between the auxiliary planner's problem, which we have just shown has a recursive structure, and the planner's problem. In the planner's problem, society is assumed to know the "seed value" $h_{-1}$ of the endowment process, for each agent, and minimize the cost of providing an incentive compatible allocation which delivers lifetime utility $w_{0}$. The auxiliary problem additionally imposes a condition that the allocation delivers a lifetime utility $\hat{w}_{0}$ to an agent with seed value $h_{-1}^{c}$. The fact that the auxiliary planner's problem is recursive, along with the fact that the solution to the planner's problem is also the solution to the auxiliary planner's problem for some $\hat{w}_{0}$ (equation (6)), implies that the continuation of a solution to the planner's problem is a solution to the auxiliary planner's problem from that date on, for the appropriate values of $\left(h_{-}, w, \hat{w}\right)$. This does not imply that the continuation of a solution to the planner's problem is itself a solution to the planner's problem for the appropriate $\left(h_{-1}, w\right)$, which is precisely the condition needed for a plan to be ex-post efficient.

In the recursive form of the auxiliary planning problem, there are constraints which would not exist in any ex-post Pareto optimization. Specifically, on the equilibrium path, the agent who lied last period about his endowment doesn't exist, and keeping this agent's utility constant (equation (23)) is simply an extra constraint. If this constraint binds, by reoptimizing without the constraint both parties could be made better off, implying that a solution to the planner's problem is not "time-consistent." 


\section{COMPUTATION AND CHARACTERIZATION}

The recursive formulation given is itself an algorithm for computing efficient contracts. The set $W\left(h_{-}\right)$(for each $h_{-}$) can be computed as follows. First, the highest $w$ which can be delivered is simply $U(\bar{b}) /(1-\beta)$. Next, the lowest $w$ which can be delivered is the utility associated with consuming $\underline{b}$ whenever $h=h_{L}$ and consuming $\underline{b}+h_{H}-h_{L}$ whenever $h=h_{H}$. (This utility can always be achieved unilaterally by an agent.) Let $\underline{w}(h)$ denote the lowest discounted utility attainable when $h$ occurred last period. Thus the set $W\left(h_{-}\right)$can be represented by two functions defining the highest and lowest $\hat{w}$ which can be delivered for each $\left(h_{-}, w\right), h \in H$ and $w \in[\underline{w}(h), \bar{d}]$. If these functions are set to the highest and lowest values associated with bounded consumption, repeated application of the APS operator outlined in the proof of Lemma 2.2 converges to the true set $W^{*}\left(h_{-}\right)$. Given $W^{*}\left(h_{-}\right)$, the function $V_{A}\left(h_{-}, w, \hat{w}\right)$ can be computed using the $T$ operator defined in equation (21).

We have computed the following example. An agent's endowment is either $h_{L}=.1$ or $h_{H}=.35$. We assume that an agent who has endowment $h_{H}$ can claim $h_{L}$ but an agent with endowment $h_{L}$ cannot claim $h_{H}$. (See footnote 3.) The Markov process governing these endowments is simply that the agent has a $90 \%$ chance of receiving the endowment he received in the previous period. His utility function is $U(c)=\sqrt{c}$ with a restriction that $c \in[0,1]$ with a discount parameter $\beta=.9$.

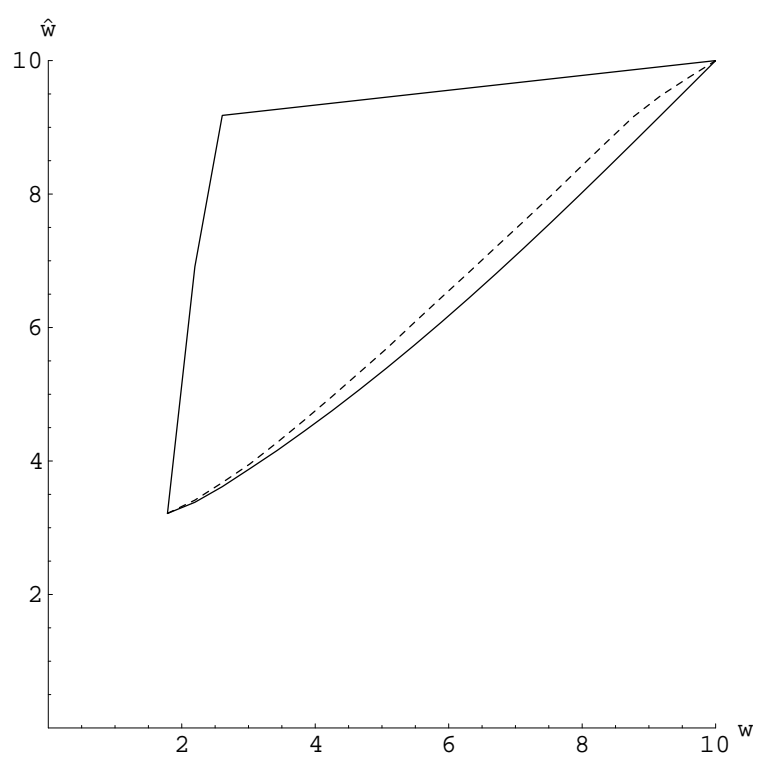

Figure 1: $W^{*}\left(h_{L}\right)$ 
Figure 1 displays the set of feasible utility pairs $(w, \hat{w})$ given $h_{-}=h_{L}$, $W^{*}\left(h_{L}\right)$. The dashed line represents the $\hat{w}$ point which achieves the minimum of $V_{A}\left(h_{L}, w, \hat{w}\right.$ ) (or the ex-post efficient $\hat{w}$ given $w$ ). Call this function $\hat{w}^{*}(w)$. The area above $\hat{w}^{*}(w)$ is, in an important sense, irrelevant. Examination of the Bellman equation (21) reveals that no efficient $t=0$ contract would ever map to this area. Doing so both raises costs and tightens the incentive constraint at $t=0$. (Since $h_{H}$ cannot be falsely claimed, this graph is unnecessary for $h_{-}=h_{H}$. If an agent announces $h_{H}$, the continuation contract is always ex-post efficient since the type of agent is common knowledge.) The area below $\hat{w}^{*}(w)$ represents the ex-post inefficient points that may be mapped to by the continuation of an efficient contract. Doing so loosens the incentive constraint at period $t=0$. In fact, if an efficient contract maps to $w^{\prime}\left(h_{L}\right)$ and $V_{A}\left(h_{L}, w^{\prime}\left(h_{L}\right), \hat{w}^{*}\left(w^{\prime}\left(h_{L}\right)\right)\right)$ is differentiable with respect to $\hat{w}$ at this point, then the continuation given $h=h_{L}$ must be inefficient. (The $t=0$ incentive constraint is loosened at zero marginal cost). We have not proved that $V_{A}$ is differentiable. On the other hand, Figure 2 displays $V_{A}\left(h_{L}, w, \hat{w}\right)$ for a particular $w$ for this example and it appears smooth.

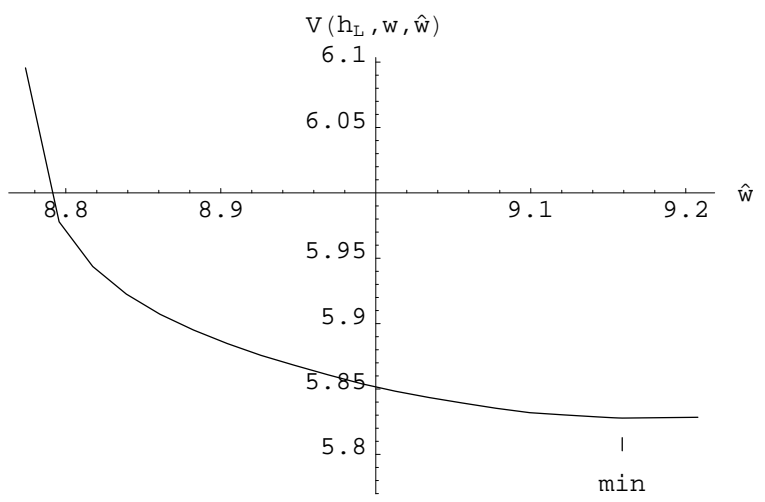

Figure 2: $V_{A}\left(h_{L}, w=8.75, \hat{w}\right)$.

What do such inefficient contracts look like? For our computed example, two characteristics stand out. First, the continuation of an inefficient contract is itself an inefficient contract as long as $h_{t}=h_{L}$, but less so each time. This is shown in Figure 3 which displays the path of $\left(w_{t}, \hat{w}_{t}\right)$ when $h_{-}=h_{H}$, but then a long string of $h_{L}$ realizations occurs. 


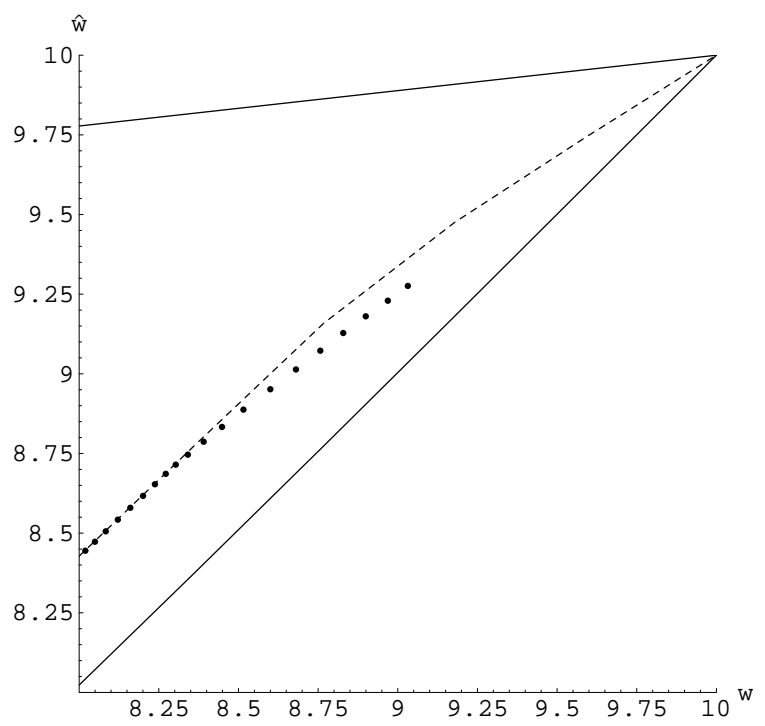

Figure 3: Path of $(w, \hat{w})$ given string of $h_{L}$ realizations.

Second, inefficient contracts given $h_{-}=h_{L}$ exaggerate a property of expost efficient contracts of pushing utility payments toward current utility payments conditional on the low realization, $u\left(h_{L}\right)$. That is, relative to the autarkic contract (zero transfers at all times) an efficient incentive compatible contract pushes utility payments toward the low realization for insurance reasons. To do so in a way which holds $w$ constant, the contract must raise the sum $u\left(h_{L}\right)+\beta w^{\prime}\left(h_{L}\right)$ and lower the sum $u\left(h_{H}\right)+\beta w^{\prime}\left(h_{H}\right)$ by a given proportion. This also lowers the left hand side (truth-telling) of the incentive constraint associated with $h=h_{H}$. Maintaining incentive compatibility then requires that the right hand side (the value to falsely claiming low) be decreased even though the utility of an agent truthfully announcing low, $u\left(h_{L}\right)+\beta w^{\prime}\left(h_{L}\right)$, is being increased. This is achieved by exploiting the fact that an agent who actually has an endowment of $h_{L}$ values current marginal consumption more than an agent who is falsely announcing $h_{L}$. That is, $u\left(h_{L}\right)$ is raised and both $w^{\prime}\left(h_{L}\right)$ and $\hat{w}^{\prime}\left(h_{L}\right)$ are lowered relative to the autarkic contract. The ex-post efficient contact, in effect, optimally trades the insurance benefit associated with pushing utility toward the $h=h_{L}$ realization, and the cost associated with uneven consumption over time needed to make this insurance incentive compatible. The ex-post inefficient contracts act much in the same way, but where more utility is pushed toward the $h=h_{L}$ realization than the insurance motive alone would justify. 


\section{EXTENSIONS AND OTHER ENVIRONMENTS}

An important issue is whether the methodology we present for the stochastic endowment economy with history dependent shocks is general. For instance, we require the shock to take on only two values. If the shock instead were to take on $N$ values, our state variables would be $\left(h_{-1}, w, \hat{\mathbf{w}}\right)$, where $\hat{\mathbf{w}}$ is an $N-1$ element vector specifying the continuation utility for each possible realization of $h$ in the previous period other than the report $h_{-1}$. Likewise, if the Markov process is of finite order $N>1$, it is well known that this can be mapped into a single stage Markov process where the state space $h$ is expanded to include the last $N$ realizations of the endowment. In this case, our state variables are again $\left(h_{-1}, w, \hat{\mathbf{w}}\right)$, but where $h_{-1}$ takes on $2^{N}$ values and $\hat{\mathbf{w}}$ is a $2^{N}-1$ element vector. In general, what is required is that the state variables contain the utility of every possible unobserved "type" at the beginning of period $t$. Two agents are of a different type at the beginning of a period if there is any continuation contract and continuation strategy where the utilities of the two agents differ given the continuation contract and strategy.

Another relevant matter concerns the applicability of lemma 2.1. The endowment shock environment presented above displays a form of separability in the sense that past reports do not affect one's incentives to report truthfully from today onwards. In other words, truth-telling incentives for the current date on are effective concerning both an agent who misreported the shock realization in the past as well as a truth-telling agent. In other environments, however, it is possible that after previous deviations, the agent may prefer to engage in a strategy of infinite deviations. In fact, this non-separability is present in the privately observed effort model discussed below which, nonetheless, has a recursive structure.

Besanko [3] characterizes a multi-period contract between principal and agent in the presence of adverse selection, under the assumption that principal and agent are risk-neutral. In his model, the privately observed costefficiency parameter can take on a continuum of values. He derives the recursive form of the incentive compatibility constraint for his problem, the analogue of equation (13) in our paper. The structure of this class of models enables direct substitution of this period by period condition in the principal's sequence utility function. Direct substitution provides an additional simplification in that threat keeping is automatically imposed as well. Since past shocks do not affect current utility directly, his problem displays the separability property referred to, above, indicating that truth-telling incentives to an agent who has reported truthfully also apply to an agent who misreported his type in the past. Confronting Besanko's result with those in our paper, we were able to generalize the incentive compatibility conditions to non-separable situations (see section 4.2), as 
well as provide a complete recursive formulation of the infinite sequence contract. The recursive formulation presented here is not well-suited to handle a continuum of types, however.

We next apply the recursive formulation to two other environments commonly seen in the repeated contracting literature: unobserved taste shocks (the focus of Atkeson and Lucas [2]) and unobserved effort (the focus of Spear and Srivastava [11] and Phelan and Townsend [10]). In each of these environments, the authors have assumed that privately observed random variables are independent over time, and privately observed efforts only affect output in the period the effort is taken. These assumptions precisely insure that there is only one type at the beginning of any period. (Past deviations do not affect one's utility for any continuation contract given any strategy.)

In the introduction, we argued for the relevance of relaxing these assumptions. We now move to demonstrating the recursive structure for a taste shock model with Markov shocks and an unobserved effort model where output depends on effort in the previous period as well as on the current effort choice. We show analogues of Theorem 1 for both these environments and produce the corresponding recursive formulations.

We note that these two environments differ in one respect, however. For the case of correlated taste shocks, an analogue of Lemma 1 holds, therefore ensuring that incentive compatible allocations (from the perspective of time 0) are also incentive compatible at any later date, after all histories of reports and realizations of the shocks. In the effort model, where output depends on current and last period's effort, this separability is no longer present: if the agent took an action different than the recommended level of effort in the past, he may prefer to continue to deviate from the recommended actions today and in the future. We show how to modify the promised utility for a deviant agent in order to accommodate this non-separability, and point out how to interpret "temporary incentive compatibility," in this context.

\subsection{Private Taste Shocks}

The private taste shock model can be described by making a few simple changes to the privately observed endowment model. First, let each agent's endowment always equal $\bar{e}$. Next, as before, let $h_{t} \in\left\{h_{L}, h_{H}\right\}$ with $\Pi\left(h^{t} \mid h_{0}\right)$ denoting the probability of history $h^{t}=\left\{h_{0}, h_{1}, \ldots, h_{t}\right\}$ given $h_{0}$, but change the interpretation of $h_{t}$ from an endowment shock to a taste shock. That is, if $c=\left\{c_{t}\right\}_{t=0}^{\infty}$ and $h=\left\{h_{t}\right\}_{t=0}^{\infty}$ are deterministic sequences of consumption and taste shocks, respectively, let

$$
\bar{U}(c, h)=\sum_{t=0}^{\infty} \beta^{t} U\left(c_{t}, h_{t}\right),
$$


where $U: B \times H \rightarrow R$ is strictly increasing and strictly concave in its first argument and $\beta \in(0,1)$. Feasible values for discounted utility are then $D_{h}=\left[\underline{d}_{h}, \bar{d}_{h}\right]$, where $\underline{d}_{h}=U(\underline{b}, h) /(1-\beta)$ and $\bar{d}_{h}=U(\bar{b}, h) /(1-\beta)$ for $h \in\left\{h_{L}, h_{H}\right\}$.

As before, we can consider the planner to be delivering utils as opposed to consumption transfers. Where before the amount of the consumption needed to deliver $\tilde{u}$ utils was $C(\tilde{u})=U^{-1}(\tilde{u})$, here the amount of the consumption needed to deliver $\tilde{u}$ utils is $C(\tilde{u}, h)=U^{-1}(\tilde{u}, h)$. This structure is so similar to the unobserved endowment model that Lemma 1 and Theorem 1 go through with little modification.

Given this, we move to writing the auxiliary planner's problem in recursive form. As before, let $W^{*}\left(h_{-}\right)$denote the set of $(w, \hat{w})$ pairs such that there exists an allocation satisfying (the current analogues of) promise keeping, threat keeping and incentive compatibility. For each $h \in H$, the choice variables are $u(h)$, the current utility transfer as a function of the announced shock; $w^{\prime}(h)$, the lifetime continuation utility promise as a function of the announced shock; and $\hat{w}^{\prime}(h)$, the utility that the planner tells the agent he will receive from the continuation allocation if he announces $h$ but is lying. These are exactly the same choice variables for the recursive form of the unobserved endowment model. The state variables $-h_{-}$, the reported shock last period; $w$, the promised utility of an agent who truthfully announced $h_{-}$last period; and $\hat{w}$, the promised utility of an agent who falsely announced $h_{-}$last period - are the same as for the unobserved endowment model as well. The recursive operator $T$, defined on the space of bounded continuous functions mapping $H \times D \times D \rightarrow R$, is then

$$
\begin{gathered}
T\left(v_{A}\right)\left(h_{-}, w, \hat{w}\right)= \\
\min _{u(h), w^{\prime}(h), \hat{w}^{\prime}(h)} \sum_{h \in H}\left\{C(u(h), h)-\bar{e}+q v_{A}\left(h, w^{\prime}(h), \hat{w}^{\prime}(h)\right)\right\} \Pi\left(h \mid h_{-}\right)
\end{gathered}
$$

subject to

$$
\begin{aligned}
& w=\sum_{h \in H}\left\{u(h)+\beta w^{\prime}(h)\right\} \Pi\left(h \mid h_{-}\right), \\
& \hat{w}=\sum_{h \in H}\left\{u(h)+\beta w^{\prime}(h)\right\} \Pi\left(h \mid h_{-}^{c}\right)
\end{aligned}
$$

and, for each $h \in H$,

$$
u(h)+\beta w^{\prime}(h) \geq U\left(C\left(u\left(h^{c}\right), h^{c}\right), h\right)+\beta \hat{w}^{\prime}\left(h^{c}\right),
$$

as well as $u(h) \in D_{h}$ and $\left(w^{\prime}(h), \hat{w}^{\prime}(h)\right) \in W^{*}(h)$. 


\subsection{Privately Observed Effort}

In this section we consider a privately observed effort model where output depends on effort in the previous period as well as on the current effort choice. This environment is similar to the previous two models in that at the beginning of date $t$, the "type" of agent is not common knowledge: the agent knows how much he worked in the previous period while the planner does not. As in the previous environments, and unlike environments where output only depends on effort in the current period, this eliminates common knowledge regarding the probability of future events. The novelty of the current environment, however, is that unlike the endowment and taste shock cases, in the current setup no analogue of Lemma 1 holds: there is no guarantee that an incentive compatible plan will have an agent who deviated in the past not wishing to deviate in the future. Nevertheless, we show that an analogue to Theorem 1 holds.

Here, let $h_{t} \in\left\{h_{L}, h_{H}\right\}$ denote the observable endowment. Next, let the probability of the high endowment in period $t$, denoted $\pi\left(a_{t}, a_{t-1}\right)$, depend on unobserved effort $a \in A=\left\{a_{L}, a_{H}\right\}$ carried out by the agent in the current and previous periods. This specifies the physical environment. Next, assume agents evaluate certain paths of consumption and effort according to

$$
\bar{U}(c, a)=\sum_{t=0}^{\infty} \beta^{t} U\left(c_{t}, a_{t}\right),
$$

where $U: B \times A \rightarrow R$ is strictly increasing and strictly concave in its first argument and $\beta \in(0,1)$. Feasible values of momentary utility are then $D_{a}=\left[\underline{d}_{a}, \bar{d}_{a}\right]$, where $\underline{d}_{a}=U(\underline{b}, a) /(1-\beta)$ and $\bar{d}_{a}=U(\bar{b}, a) /(1-\beta)$ for $a \in A$.

As in the previous two environments, we can consider the planner to be delivering utils as opposed to consumption transfers. Here the amount of consumption necessary to deliver $\tilde{u}$ utils is $C(\tilde{u}, a)=U^{-1}(\tilde{u}, a)$. We define an effort strategy as a sequence $\widetilde{a}=\left\{\widetilde{a}_{t}\left(h^{t-1}\right)\right\}_{t=0}^{\infty}$ where $\widetilde{a}_{t}: H^{t-1} \rightarrow A$. Next, recall that in the auxiliary planner's problem for the unobserved endowment environment, the planner not only must deliver a specified lifetime utility $w_{0}$ to an agent whose "seed" value of the Markov process is $h_{-1}$ but also must deliver a lifetime utility $\hat{w}_{0}$ to an agent whose seed value is $h_{-1}^{c}$. In this case, we require this as well, where these seed values correspond to $a_{-1}$ and $a_{-1}^{c}$. (In this environment, it is actions, not the endowments, which are unobserved.)

We define an allocation, then, as a pair of sequences of functions $a=$ $\left\{a_{t}\left(h^{t-1}\right)\right\}_{t=0}^{\infty}$, describing the recommended efforts for the agent, and $u=$ $\left\{u_{t}\left(h^{t}\right)\right\}_{t=0}^{\infty}$ where $u_{t}: H^{t} \rightarrow D_{a_{t}\left(h^{t-1}\right)}$, describing utility payments.

Let $\Pi\left(h^{t} \mid a, a_{-1}\right)$ denote the probability of sequence $\left\{h_{0}, h_{1}, \ldots, h_{t}\right\}$ given $\pi$, an effort strategy $a$ and a seed value $a_{-1}$. We say an allocation is incen- 
tive compatible if it induces an agent to follow the recommended strategy. That is, an incentive compatible allocation $\{a, u\}$ satisfies, for all effort strategies $\widetilde{a}$,

$$
\begin{gathered}
\sum_{t=0}^{\infty} \sum_{h^{t} \in H^{t}} \beta^{t} u_{t}\left(h^{t}\right) \Pi\left(h^{t} \mid a, a_{-1}\right) \\
\geq \sum_{t=0}^{\infty} \sum_{h^{t} \in H^{t}} \beta^{t} U\left(C\left(u_{t}\left(h^{t}\right), a_{t}\left(h^{t-1}\right)\right), \tilde{a}_{t}\left(h^{t-1}\right)\right) \Pi\left(h^{t} \mid \widetilde{a}, a_{-1}\right) .
\end{gathered}
$$

An allocation satisfies promise keeping if it delivers $w_{0}$ units of expected discounted utility to an agent with seed value $a_{-1}$ :

$$
w_{0}=\sum_{t=0}^{\infty} \sum_{h^{t} \in H^{t}} \beta^{t} u_{t}\left(h^{t}\right) \Pi\left(h^{t} \mid a, a_{-1}\right)
$$

An allocation satisfies threat keeping if it delivers $\hat{w}_{0}$ units of expected discounted utility to an optimizing agent with seed value $a_{-1}^{c}$ :

$$
\hat{w}_{0}=\max _{\widetilde{a}} \sum_{t=0}^{\infty} \sum_{h^{t} \in H^{t}} \beta^{t} U\left(C\left(u_{t}\left(h^{t}\right), a_{t}\left(h^{t-1}\right)\right), \widetilde{a}_{t}\left(h^{t-1}\right)\right) \Pi\left(h^{t} \mid \widetilde{a}, a_{-1}^{c}\right) .
$$

Let $W^{*}\left(a_{-}\right)$be the set of $(w, \hat{w})$ pairs such that there exists an allocation satisfying $(30),(31)$ and $(32)$. Given initial state variables $\left(a_{-1}, w_{0}, \hat{w}_{0}\right) \in$ $A \times W^{*}\left(a_{-1}\right)$, the auxiliary planner's problem is defined as choosing an allocation $\{a, u\}$ to minimize the cost of providing $\left(w_{0}, \hat{w}_{0}\right)$ units of expected discounted utility:

$$
V_{A}\left(a_{-1}, w_{0}, \hat{w}_{0}\right)=\min _{a, u}\left\{\sum_{t=0}^{\infty} \sum_{h^{t} \in H^{t}} q^{t}\left[C\left(u_{t}\left(h^{t}\right)\right)-h_{t}\right] \Pi\left(h^{t} \mid a, a_{-1}\right)\right\}
$$

subject to (30) through (32).

Next, take as given an allocation $\{a, u\}$. Let $a\left(h^{t-1}\right)=\left\{a_{\tau}\left(h^{\tau-1} \mid\right.\right.$ $\left.\left.h^{t-1}\right)\right\}_{\tau=0}^{\infty}$ denote the continuation strategy induced by $a$ after endowment realization $h^{t-1}$. That is, $a_{\tau}\left(h^{\tau-1} \mid h^{t-1}\right)=a_{t+\tau}\left(h^{t+\tau-1}\right)$. For such a plan, let

$$
w_{t}\left(h^{t-1}\right)=\sum_{\tau=0}^{\infty} \sum_{h^{\tau} \in H^{\tau}} \beta^{\tau} u_{t+\tau}\left(h^{t-1}, h^{\tau}\right) \Pi\left(h^{\tau} \mid a\left(h^{t-1}\right), a_{t-1}\left(h^{t-2}\right)\right)
$$


This represents the forward looking utility of an obedient agent up to and after date $t$. Equation (34) can be written recursively as

$$
\begin{gathered}
w_{t}\left(h^{t-1}\right)=\sum_{h_{t} \in H}\left(u_{t}\left(h^{t-1}, h_{t}\right)\right. \\
\left.+\beta w_{t+1}\left(h^{t-1}, h_{t}\right)\right) \pi\left(h_{t} \mid a_{t}\left(h^{t-1}\right), a_{t-1}\left(h^{t-2}\right)\right) .
\end{gathered}
$$

Let

$$
\begin{gathered}
\hat{w}_{t}\left(h^{t-1}\right)=\max _{\widetilde{a}} \sum_{\tau=0}^{\infty} \sum_{h^{\tau} \in H^{\tau}} \beta^{\tau} U\left(C \left(u_{t+\tau}\left(h^{t-1}, h^{\tau}\right),\right.\right. \\
\left.\left.a_{t+\tau}\left(h^{t-1}, h^{\tau-1}\right)\right), \widetilde{a}_{\tau}\left(h^{t-1}, h^{\tau-1}\right)\right) \Pi\left(h^{\tau} \mid \widetilde{a}\left(h^{t-1}\right), a_{t}^{c}\left(h^{t-2}\right)\right) .
\end{gathered}
$$

This represents the forward looking utility of an optimizing agent who obeyed up to date $t-2$ but deviated at date $t-1$. This can also be written recursively as

$$
\begin{gathered}
\hat{w}_{t}\left(h^{t-1}\right)= \\
\max \left\{\sum_{h_{t} \in H}\left(u_{t}\left(h^{t-1}, h_{t}\right)+\beta w_{t+1}\left(h^{t-1}, h_{t}\right)\right) \pi\left(h_{t} \mid a_{t}\left(h^{t-1}\right), a_{t-1}^{c}\left(h^{t-2}\right)\right),\right. \\
\sum_{h_{t} \in H}\left(U\left(C\left(u_{t}\left(h^{t-1}, h_{t}\right), a_{t}\left(h^{t-1}\right)\right), a_{t}^{c}\left(h^{t-1}\right)\right)\right. \\
\left.\left.+\beta \hat{w}_{t+1}\left(h^{t-1}, h_{t}\right)\right) \pi\left(h_{t} \mid a_{t}^{c}\left(h^{t-1}\right), a_{t-1}^{c}\left(h^{t-2}\right)\right)\right\} .
\end{gathered}
$$

We now prove the analogue of Theorem 1 .

THEOREM 4.1. The allocation $\{a, u\}$ is incentive compatible (satisfies (30)) if and only if it satisfies the following condition, for all time periods $t-1$ and all histories $h^{t-1}$ :

$$
\begin{gathered}
\sum_{h_{t}}\left\{u_{t}\left(h^{t-1}, h_{t}\right)+\beta w_{t+1}\left(h^{t-1}, h_{t}\right)\right\} \\
\Pi\left(h_{t} \mid a_{t}\left(h^{t-1}\right), a_{t-1}\left(h^{t-2}\right)\right) \geq \\
\sum_{h_{t}}\left\{U\left(C\left(u_{t}\left(h^{t-1}, h_{t}\right), a_{t}\left(h^{t-1}\right)\right), a_{t}^{c}\left(h^{t-1}\right)\right)+\beta \hat{w}_{t+1}\left(h^{t-1}, h_{t}\right)\right\}
\end{gathered}
$$




$$
\Pi\left(h_{t} \mid a_{t}^{c}\left(h^{t-1}\right), a_{t-1}\left(h^{t-2}\right)\right) .
$$

Proof. An incentive compatible allocation trivially satisfies (38). We must show that, if $\{a, u\}$ satisfies (38), it satisfies (30). Suppose not. Then, for a specification of $\{a, u\}$ that satisfies (38), there exists an effort strategy $\widetilde{a}$ such that

$$
\begin{gathered}
W_{0}(\widetilde{a}) \equiv \\
\sum_{t=0}^{\infty} \sum_{h^{t} \in H^{t}} \beta^{t} U\left(C\left(u_{t}\left(h^{t}\right), a_{t}\left(h^{t-1}\right)\right), \widetilde{a}_{t}\left(h^{t-1}\right)\right) \Pi\left(h^{t} \mid \widetilde{a}, a_{-1}\right)
\end{gathered}
$$

exceeds $w_{0}$, the utility associated with obedience.

Let $\tau^{*}$ denote the first date where there exists some history $h^{\tau^{*}-1}$ where action $a_{\tau}\left(h^{\tau^{*}-1}\right) \neq \widetilde{a}_{\tau}\left(h^{\tau^{*}-1}\right)$. Consider equation (38), for an arbitrary $h^{\tau^{*}-1}$. Here, the left hand side is the continuation utility of following the recommended action strategy $a_{\tau}\left(h^{\tau^{*}-1}\right)$. The right hand side is the continuation utility of taking action $a_{\tau}^{c}\left(h^{\tau^{*}-1}\right)$ and following the best deviation strategy from $\tau^{*}+1$ on. Thus the left hand side weakly exceeds the continuation utility of following $\widetilde{a}$. Since the per period utilities of following $a$ and $\widetilde{a}$ are equal for dates $t<\tau^{*}$ and the above holds for all histories $h^{\tau^{*}-1}$, $W_{0}(\widetilde{a}) \leq w_{0}$, yielding a contradiction.

This theorem then implies that the incentive constraint (38) can be written recursively as

$$
\begin{gathered}
w_{t}\left(h^{t-1}\right) \geq \sum_{h_{t}}\left\{U\left(C\left(u_{t}\left(h^{t-1}, h_{t}\right), a_{t}\left(h^{t-1}\right)\right), a_{t}^{c}\left(h^{t-1}\right)\right)+\right. \\
\left.\beta \hat{w}_{t+1}\left(h^{t-1}, h_{t}\right)\right\} \pi\left(h_{t} \mid a_{t}^{c}\left(h^{t-1}\right), a_{t-1}\left(h^{t-2}\right)\right),
\end{gathered}
$$

given equations (35) and (36).

We note that the fact that Lemma 1 does not hold in this environment modifies the definition of the promised future utility to a deviant agent, $\hat{w}_{t}\left(h^{t-1}\right)$. In fact, the continuation utility promised to an agent who does not take the recommended action takes into account the fact that this agent may optimally choose to continue to deviate in the future. Hence, the formula for $\hat{w}_{t}\left(h^{t-1}\right)$, equation $(36)$, contains the "max" operator with respect to all possible effort strategies, no longer assuming that this agent will choose to take the recommended actions from period $t$ on. Therefore, in environments where there exists such non-separability, temporary incentive compatibility should be taken to mean that incentives are conveyed towards 
inducing truthful revelation (or adequate action) in the current period for agents who did not deviate in the past. It no longer means (as it is usually the case in the literature) that we are considering this one time deviation and going back to truth-telling, as past deviations may now induce the agent to engage in a strategy with infinite deviations.

That each constraint can be written recursively allows us to write the auxiliary planner's problem in this environment recursively, as well. In this form, the choice variables are $a$, the recommended action for today; $u(h)$, the current utility payment as a function of today's output $h ; w^{\prime}(h)$, the continuation utility of an agent who takes action $a$ in the current period and has output realization $h$; and $\hat{w}^{\prime}(h)$, the continuation utility of an agent who takes action $a^{c}$ in the current period and has output realization $h$. The state variables are then $a_{-}$, the recommended action in the previous period; $w$, the continuation utility of an agent who actually took action $a_{-}$in the previous period; and $\hat{w}$, the continuation utility of an agent who actually took action $a_{-}^{c}$ in the previous period. This makes the Bellman operator for the auxiliary programming problem in this environment

$$
\begin{gathered}
T\left(v_{A}\right)\left(a_{-}, w, \hat{w}\right)=\min _{a, u(h), w^{\prime}(h), \hat{w}^{\prime}(h)} \\
\sum_{h}\left\{C(u(h), a)-h+q v_{A}\left(a, w^{\prime}(h), \hat{w}^{\prime}(h)\right)\right\} \pi\left(h \mid a, a_{-}\right),
\end{gathered}
$$

subject to

$$
\begin{gathered}
w=\sum_{h}\left\{u(h)+\beta w^{\prime}(h)\right\} \pi\left(h \mid a, a_{-}\right) \\
\hat{w}=\max \left\{\sum_{h}\left\{u(h)+\beta w^{\prime}(h)\right\} \pi\left(h \mid a, a_{-}^{c}\right),\right. \\
\left.\left.\sum_{h}\left\{U(C(u(h), a)), a^{c}\right)+\beta \hat{w}^{\prime}(h)\right\} \pi\left(h \mid a^{c}, a_{-}^{c}\right)\right\}
\end{gathered}
$$

and

$$
\left.w \geq \sum_{h}\left\{U(C(u(h), a)), a^{c}\right)+\beta \hat{w}^{\prime}(h)\right\} \pi\left(h \mid a^{c}, a_{-}\right),
$$

as well as $u(h) \in D_{a}$ and $\left(w^{\prime}(h), \hat{w}^{\prime}(h)\right) \in W^{*}(h)$. 


\section{CONCLUSION}

In this paper, we have derived recursive methods to handle problems where privately observed variables are linked over time, an extension of agency theory to more realistic environments. We have used Green's [6] model with correlated endowment shocks as an illustrative benchmark and extended these methods to two other environments, considering both serially correlated private taste shocks as well as output as a function of privately observed current and past effort levels.

Despite our use of concrete applications, we believe the general idea underlying this recursive methodology should be clear. Links across time periods brought about by privately observed variables cause the common knowledge over preferences concerning continuation contracts to break down. This means that the planner, say, cannot distinguish among agents who have similar past public histories yet whose privately observed past is different. These potential differences in the agents' privately observed past which are compatible with their common publicly observed history are relevant to the extent they affect the valuation of continuation contracts by the agent. Enumerating such different private histories amounts to considering all possible types of agents that the planner may be facing at a certain point in time. Incentive compatibility, in this environment, then implies that the utility of all these different types of agents (who correspond to all possible past deviations in the form of either lies or not following recommended actions) has to add up to some pre-specified amounts. Not keeping these threats would violate incentive compatibility. Yet, keeping these additional constraints is Pareto inefficient since it concerns incentives applying to actions already taken in previous periods.

One of the differences of the recursive formulation presented in this paper - as compared to the common knowledge benchmark — is, then, the additional component of threat keeping. These constraints, together with the temporary incentive compatibility and promise keeping constraints, implement an infinite sequence of utility endowments which is incentive compatible from time 0 .

Another point which is specific to the presence of links across time periods is the possibility, in some applications, that agents who deviated once (either misreported income or did not take the recommended action) may want to follow a strategy of infinite future deviations. We showed how to take this non-separability into account by keeping constant the agent's future utility computed under the best possible strategy from the agent's perspective. 


\section{REFERENCES}

1. Abreu, Dilip, Pearce, David and Ennio Stacchetti (1990) "Toward a Theory of Discounted Repeated Games With Imperfect Monitoring," Econometrica, vol. 58(5), pp. 1041-1063.

2. Atkeson, Andrew and Robert E. Lucas, Jr. (1992), "On Efficient Distribution with Private Information," Review of Economic Studies, vol. 59(3), pp. 427-453.

3. Besanko, David (1985), "Multi-Period Contracts Between Principal and Agent With Adverse Selection," Economic Letters, vol. 17(1), pp. 33-37.

4. Cole, Harold L. and Narayana R. Kocherlakota (1998), "Efficient Allocations With Hidden Income and Hidden Storage," Federal Reserve Bank of Minneapolis Staff Report No. 238.

5. Cole, Harold L. and Narayana R. Kocherlakota (1998), "Dynamic Games With Hidden Actions and Hidden States," Federal Reserve Bank of Minneapolis Staff Report No. 254.

6. Green, Edward J. (1987), "Lending and the Smoothing of Uninsurable Income," in E. Prescott and N. Wallace, (Eds.), Contractual Arrangements for Intertemporal Trade (Minneapolis: University of Minnesota Press), pp. 3-25.

7. Hopenhayn, Hugo A. and Juan Pablo Nicolini (1997), "Optimal Unemployment Insurance," Journal of Political Economy, vol. 105(2), pp. 412-438.

8. Phelan, Christopher (1994), "Incentives and Aggregate Shocks," Review of Economic Studies, vol. 61(4), pp. 681-700.

9. Phelan, Christopher (1995), "Repeated Moral Hazard and One-Sided Commitment," Journal of Economic Theory, vol. 66(2), pp. 488-506.

10. Phelan, Christopher and Robert M. Townsend (1991), "Computing Multi-Period, Information-Constrained Optima," Review of Economic Studies, vol. 58(5), pp. 853881.

11. Spear, Stephen E. and Sanjay Srivastava (1987), "On Repeated Moral Hazard with Discounting," Review of Economic Studies, vol. 54(4), pp. 599-617.

12. Stokey, Nancy L. and Robert E. Lucas, Jr., with Edward C. Prescott (1989), Recursive Methods in Economic Dynamics (Cambridge, Mass: Harvard University Press).

13. Thomas, Jonathan and Tim Worrall (1990), "Income Fluctuations and Asymmetric Information: An Example of a Repeated Principal-Agent Problem," Journal of Economic Theory, vol. 51(2), pp. 367-390.

14. Taub, Bart (1998) "International Financial Equilibrium with Risk Sharing and Private Information," in Current Trends in Economics: Theory and Applications, ed by A. Alkan, C. Aliprantis, and N. Yannelis. Berlin: Springer-Verlag, 1999.

15. Wang, Cheng (1995), "Dynamic Insurance with Private Information and Balanced Budgets," Review of Economic Studies, vol. 62(4), pp. 577-595. 